University of Nebraska - Lincoln

DigitalCommons@University of Nebraska - Lincoln

Roger Kirby Publications

Research Papers in Physics and Astronomy

April 1991

\title{
Magneto-optical properties of MnBiAl thin films
}

\author{
J. X. Shen \\ University of Nebraska - Lincoln \\ Roger D. Kirby \\ University of Nebraska-Lincoln, rkirby1@unl.edu \\ David J. Sellmyer \\ University of Nebraska-Lincoln, dsellmyer@unl.edu \\ Y. J. Wang \\ Chinese Academy of Sciences, Beijing
}

Follow this and additional works at: https://digitalcommons.unl.edu/physics_kirby

Part of the Physics Commons

Shen, J. X.; Kirby, Roger D.; Sellmyer, David J.; and Wang, Y. J., "Magneto-optical properties of MnBiAl thin films" (1991). Roger Kirby Publications. 13.

https://digitalcommons.unl.edu/physics_kirby/13

This Article is brought to you for free and open access by the Research Papers in Physics and Astronomy at DigitalCommons@University of Nebraska - Lincoln. It has been accepted for inclusion in Roger Kirby Publications by an authorized administrator of DigitalCommons@University of Nebraska - Lincoln. 


\title{
Magneto-optical properties of MnBiAl thin films
}

\author{
J. X. Shen, R. D. Kirby, and D. J. Sellmyer \\ Behlen Laboratory of Physics and Center for Materials Research and Analysis, University of Nebraska, \\ Lincoln, Nebraska 68588-0111 \\ Y. J. Wang \\ Institute of Physics, Chinese Academy of Sciences, Beijing 100080, People's Republic of China
}

\begin{abstract}
$\mathrm{Mn}-\mathrm{Bi}-\mathrm{Al}$ thin films. were produced by sequential evaporation of the constituents, followed by an anneal at $300^{\circ} \mathrm{C}$. The temperature and composition dependencies of the Kerr rotation angle, absolute reflectivity, and magnetic anisotropy were measured. The results show that, up to 30 at. $\% \mathrm{Al}$ concentration, the thin films retain the pure $\mathrm{MnBi}$ hexagonal structure.

Further, for suitable Al content, the films have the same large Kerr rotation as MnBi.

Pure $\mathrm{MnBi}$ films exhibit perpendicular anisotropy at room temperature and in-plane anisotropy for temperatures below $142 \mathrm{~K}$. In contrast, the Al-doped films prepared here have perpendicular anisotropy down to at least $85 \mathrm{~K}$. The increased coercivities of the Al-doped films are attributed to the occupation of grain-boundary and interstitial sites of the NiAs-type hexagonal structure by the Al atoms.
\end{abstract}

\section{INTRODUCTION}

High-density magneto-optical storage technology requires the recording medium have a large polar Kerr rotation. Currently, the most promising magneto-optical materials, rare-earth-transition-metal amorphous thin films and $\mathrm{Pt} / \mathrm{Co}$ multilayer films, only have a polar Kerr rotation of $0.1^{\circ}-0.4^{\circ} .^{1,2}$ Although there are methods to enhance the Kerr rotation, such as dielectric antireflection coatings ${ }^{3}$ and optimization of the optical properties of the substrate, ${ }^{4}$ it is still of great interest to look for a material that possesses a large intrinsic Kerr rotation and good related physical properties.

So far, the materials with the largest Kerr rotations are certain U-based compounds, ${ }^{5} \mathrm{PtMnSb},{ }^{6}$ and $\mathrm{MnBi}^{7}$ The Curie temperatures of the $\mathrm{U}$ compounds are mostly well below room temperature, while $\mathrm{PtMnSb}$ has very weak or no perpendicular anisotropy, so that these materials are not suitable for practical application. On the other hand, $\mathrm{MnBi}$ has a large Kerr rotation and very strong perpendicular anisotropy. ${ }^{7}$ Unfortunately, it is polycrystalline and has a first-order phase transition near its Curie temperature. In order to overcome these disadvantages, considerable work already has been done on $\mathrm{MnBi}$ films modified by doping with, for example, $\mathrm{Ti}^{8}{ }^{8} \mathrm{Cu},{ }^{9}$ and $\mathrm{Dy} .{ }^{10} \mathrm{In}$ some of these experiments, doping improved the thermal stability and some reduced the Curie temperature, but at the same time it reduced the Faraday or Kerr rotation as well.

Recently, Wang studied the effects of ( $\mathrm{Si}, \mathrm{Al})$ doping on the MnBi thin films. ${ }^{11} \mathrm{He}$ found that ( $\mathrm{Al}, \mathrm{Si}$ ) doping increased the Kerr rotation, improved the thermal stability, and reduced the polycrystalline grain size, all important considerations for magneto-optical recording. In this paper, we will discuss the effect of $\mathrm{Al}$ on the magnetooptical properties and perpendicular anisotropy of MnBiAl thin films.

\section{EXPERIMENT}

Thin films of $\mathrm{MnBi}_{0.8} \mathrm{Al}_{x-}(0<x<1.0)$ were made by sequential evaporation of $\mathrm{Al}, \mathrm{Bi}$, and $\mathrm{Mn}$ from a tungsten boat onto a room-temperature glass substrate. The base pressure of the vacuum chamber was $2 \times 10^{-7}$ Torr, and was $3 \times 10^{-6}$ Torr during evaporation. The film thickness was typically $800 \AA$. After evaporation, the thin films were covered with an $\mathrm{SiO}$ antireflection coating of thickness $1500 \AA$ (suitable for $632.8 \mathrm{~nm}$ ). All of the samples were subsequently annealed in vacuum at about $300^{\circ} \mathrm{C}$ for at least $4 \mathrm{~h}$. X-ray-diffraction measurements confirmed that all the samples with $x<1.0$ retained the NiAs-type hexagonal structure of pure $\mathrm{MnBi}$, with the $c$ axis being highly oriented along the film normal direction. The lattice parameter is the same as that of $\mathrm{MnBi}$ within our measurement error for all the samples. As $x$ was increased above 1.0, the hexagonal structure gradually disappeared and the films became amorphous. The Kerr rotation was measured using apparatus described clscwhere. ${ }^{12}$ The tempcrature dependencies of the properties were measured using a dewar that can be used over the temperature range 80 to $600 \mathrm{~K}$. The maximum magnetic field in this measurement was $8 \mathrm{kOe}$.

\section{RESULTS AND DISCUSSION}

Figure 1 shows the $\mathrm{Al}$ concentration dependence of the Kerr rotation at room temperature. The Kerr rotation first decreases with increasing $\mathrm{Al}$ concentration, then increases for further $\mathrm{Al}$ doping. At about $x=0.9$ the Kerr rotation reaches $3.15^{\circ}$, the same value as that of $\mathrm{MnBi}_{0.8}$. The thickness of the SiO overcoat is the same for all the samples, so this phenomenon is not due to the coating. Figures 2 and 3 show the saturation Kerr rotation and absolute reflectivity wavelength dependencies of $\mathrm{MnBi}_{0.8}, \mathrm{MnBi}_{0.8} \mathrm{Al}_{0.5}$, and $\mathrm{MnBi}_{0.8} \mathrm{Al}_{0.8}$, respectively. The reflectivity oscillates with wavelength because of interference in the $\mathrm{SiO}$ overcoating. Besides the oscillation, there is little structure between 300 and $800 \mathrm{~nm}$. The Kerr rotation has a broad peak around $640 \mathrm{~nm}$ for all the samples, due mostly to the enhancement caused by the antireflection coating. The wavelength dependencies of the Kerr rotation of $\mathrm{MnBi}_{0.8}$ and $\mathrm{MnBiAl}$ have similar shapes, suggesting that the Al doping does not 


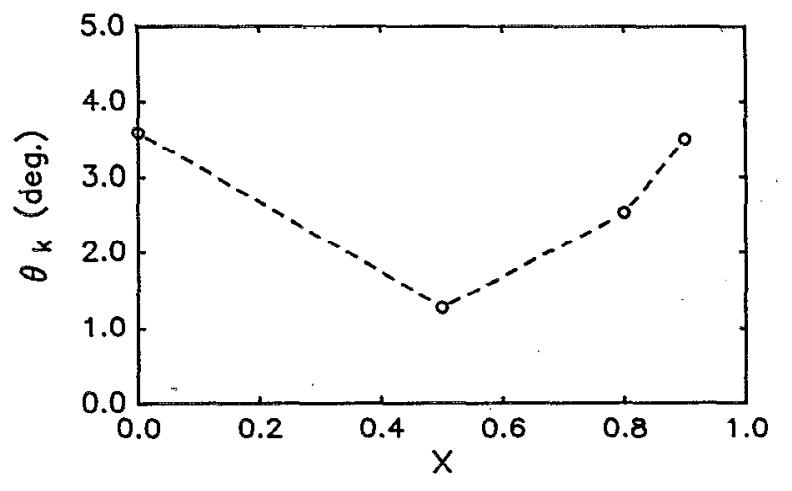

FIG. 1. Al concentration dependence of saturation Kerr rotation of $\mathrm{MnBi}_{0.8} \mathrm{Al}_{x}$ thin films at room temperature.

give rise to any additional transitions in the visible and near-infrared regions. Because of the overcoating, it is difficult to determine the intrinsic Kerr rotation for the $\mathrm{MnBiAl}$ films, but the intrinsic saturation Kerr rotation of the $\mathrm{MnBi}$ at room temperature is about $0.7^{\circ}$, so it is reasonable to estimate that the intrinsic Kerr rotation for $\mathrm{MnBi}_{0.8} \mathrm{Al}_{0.9}$ is of similar magnitude. There is no Kerr rotation enhancement due to the Al doping while such enhancement was found in the ( $\mathrm{Al}, \mathrm{Si}$ ) doping of $\mathrm{MnBi}$ thin films. ${ }^{11}$ The difference may be due to the Si content in the earlier films, since in our MnBiAl films the overcoating is $\mathrm{SiO}$, which likely bonds tightly enough to avoid diffusing into the thin films. From the band structure of MnBi calculated by Coehoorn and de Groot, ${ }^{13}$ we can see that a little shift of the Fermi level due to Al doping in the $\mathrm{MnBiAl}$ system will not affect the interband transitions very much. In other words, we do not expect a large change of the Kerr rotation through Al doping, at least in a rigid-band model. Although there is no Kerr rotation enhancement, the Kerr rotation of the $\mathrm{MnBiAl}$ system still is larger than that of the rare-earth-transition-metal amorphous thin films.

Another purpose of doping $\mathrm{MnBi}$ films with $\mathrm{Al}$ is to reduce the polycrystalline grain size and to study how the

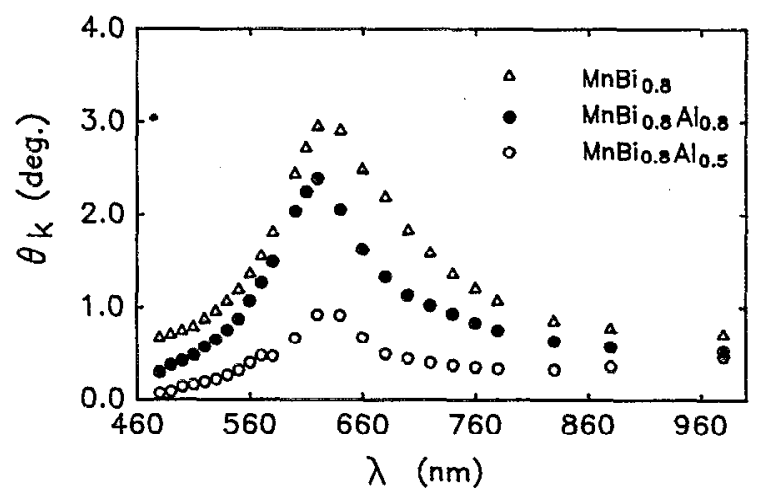

FIG. 2. Kerr rotation wavelength dependence of $\mathrm{MnBi}_{0.8}, \mathrm{MnBi}_{0.8} \mathrm{Al}_{0.5}$, and $\mathrm{MnBi}_{0.8} \mathrm{Al}_{0.8}$ films. The thickness of the $\mathrm{SiO}$ overcoat was $1500 \AA$ for $\mathrm{MnBi}_{0.8}$ and $\mathrm{MnBi}_{0.8} \mathrm{Al}_{0.8}$, and $4500 \AA$ for $\mathrm{MnBi}_{0.8} \mathrm{Al}_{0.5}$.

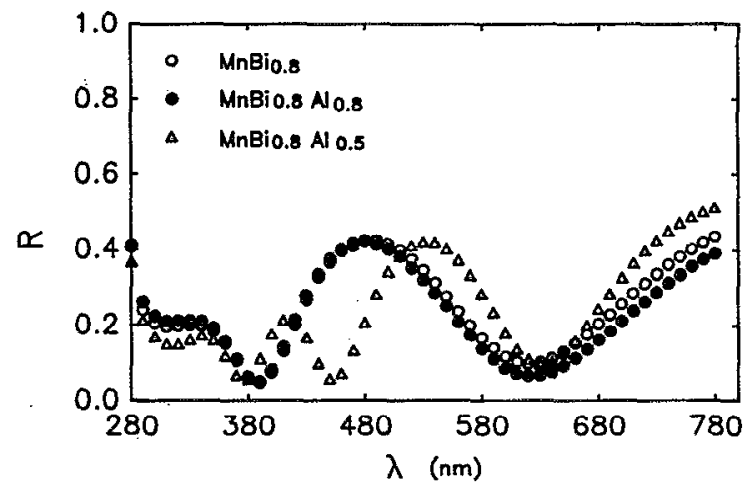

FIG. 3. Absolute reflectivity of $\mathrm{MnBi}_{0.8}, \mathrm{MnBi}_{0.8} \mathrm{Al}_{0.5}$, and $\mathrm{MnBi}_{0.8} \mathrm{Al}_{0.8}$ films.

modified microstructure affects the perpendicular anisotropy and the coercivity. Figure 4 shows Kerr hysteresis loops at $85 \mathrm{~K}$ for $\mathrm{MnBi}_{0.8}$ and $\mathrm{MnBi}_{0.8} \mathrm{Al}_{0.8}$. From the figure we can see that at that temperature, $\mathrm{MnBi}_{0.8}$ no longer has perpendicular anisotropy, in agreement with the results of Chen and Gondo. ${ }^{7}$ However $\mathrm{MnBi}_{0.8} \mathrm{Al}_{0.8}$ maintains its perpendicular anisotropy down to at least $85 \mathrm{~K}$, as is evidenced by the square hysteresis loop. Figure 5 shows the temperature dependence of the saturation and remanent Kerr rotations for $\mathrm{MnBi}_{0.8}, \mathrm{MnBi}_{0.8} \mathrm{Al}_{0.5}$, and $\mathrm{MnBi}_{0.8} \mathrm{Al}_{0.8}$. The saturation Kerr rotation of $\mathrm{MnBi}_{0.8}$ increases with decreasing temperature a little faster than those of $\mathrm{MnBi}_{0.8} \mathrm{Al}_{0.5}$ and $\mathrm{MnBi}_{0.8} \mathrm{Al}_{0.8}$, but the behaviors are qualitatively the same. We do not find such a dramatic increase (to $4.5^{\circ}$ at $80 \mathrm{~K}$ ) of the saturation Kerr rotation when the temperature lowered as was reported in Ref. 7 . However our results are in agreement with the results of the Faraday rotation measurements of Ref. 14. The increase in Kerr rotation as the temperature decreases can be attributed mostly to the increase of the saturation magnetization. The remanent Kerr rotation temperature dependence has very different features for $\mathrm{MnBi}$ and $\mathrm{MnBiAl}$ films. For the MnBiAl films, from 600 to $80 \mathrm{~K}$, the hysteresis loops are always perfectly square, meaning there is no deviation of the magnetic moment from the $c$ axis. Whereas at $T=200 \mathrm{~K}$, the remanent Kerr rotation of

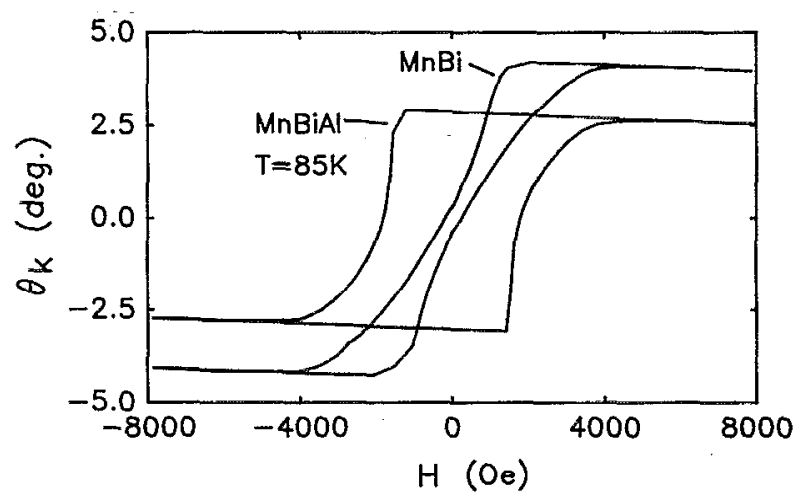

FIG. 4. Kerr hysteresis loops for $\mathrm{MnBi}_{0.8}$ and $\mathrm{MnBi}_{0.8} \mathrm{Al}_{0.8}$ films at $85 \mathrm{~K}$. 


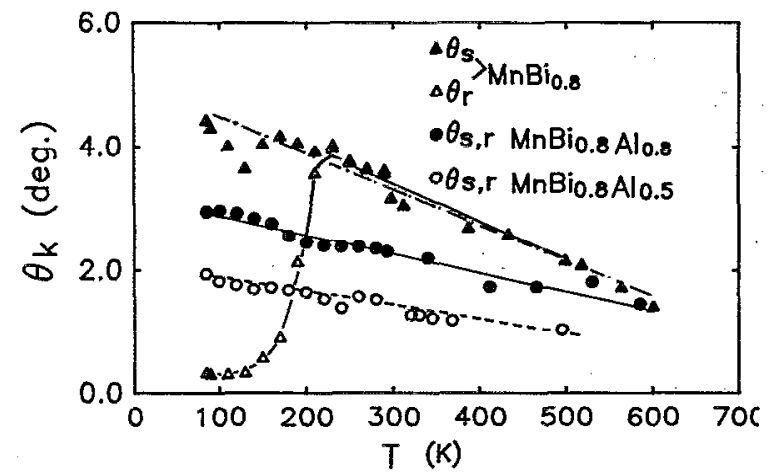

FIG. 5. Temperature dependence of the saturation and remanent Kerr rotations for $\mathrm{MnBi}_{0.8}, \mathrm{MnBi}_{0.8} \mathrm{Al}_{0.5}$, and $\mathrm{MnBi}_{0.8} \mathrm{Al}_{0.8}$ films. $\theta_{s}$ is the saturation Kerr rotation and $\theta_{r}$ is the remanent Kerr rotation.

$\mathrm{MnBi}$ gradually changes from along the $c$ axis to the basal plane. This result also was found in other experiments. ${ }^{7}$ Therefore at low temperature, the pure $\mathrm{MnBi}$ films have no perpendicular anisotropy.

Figure 6 shows the temperature dependence of the coercivity for $\mathrm{MnBi}_{0.8}, \mathrm{MnBi}_{0.8} \mathrm{Al}_{0.5}$, and $\mathrm{MnBi}_{0.8} \mathrm{Al}_{0.8}$. The coercivity increases dramatically with an increase in the $\mathrm{Al}$ concentration. The temperature dependence of the coercivity is similar for all the samples. As the temperature is increased from $85 \mathrm{~K}$, the coercivity increases with increasing temperature, presumably because of the increase in the magnetocrystalline perpendicular anisotropy. The coercivity reachcs a maximum at about $450 \mathrm{~K}$ and then decreases at higher temperature as the Curie temperature is approached.

$\mathrm{MnBi}, \mathrm{MnBiAl}$, and the related system $\mathrm{MnSb}$ all have the NiAs-type hexagonal structure. $\mathrm{MnBi}$ and $\mathrm{MnSb}$ flip their magnetic moment from along the $c$ axis into the basal plane at 142 and $510 \mathrm{~K}$, respectively. The perpendicular anisotropy in these systems presumably results from magnetocrystalline anisotropy, so that the anisotropy is closely associated with the crystal field. When the temperature decreases, the lattice will contract, which should cause a change of the local crystal field and therefore the anisotropy. When $\mathrm{MnBi}$ is doped with $\mathrm{Al}$, some $\mathrm{Al}$ atoms occupy interstitial sites of the hexagonal structure, and these atoms may block the contraction of the lattice. This may be the reason why in $\mathrm{MnBiAl}$ thin films there is no magnetic moment reorientation down to $80 \mathrm{~K}$. To test these speculations, more experimental studies are needed.

In summary, the temperature dependence of the

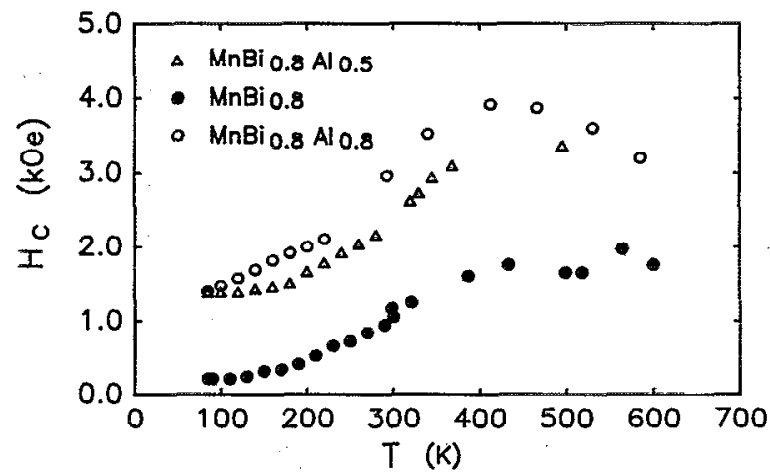

FIG. 6, Temperature dependence of the coercivities of $\mathrm{MnBi}_{0.8}$, $\mathrm{MnBi}_{0.8} \mathrm{Al}_{0.5}$, and $\mathrm{MnBi}_{0.8} \mathrm{Al}_{0.8}$ thin films.

magneto-optical and perpendicular anisotropy properties of the MnBiAl system have been investigated. Suitable Al doping does not affect the intrinsic Kerr rotation, but it changes the microstructure and anisotropy of the $\mathrm{MnBi}$ film. Between 80 and $600 \mathrm{~K}$, thin films of $\mathrm{MnBiAl}$ are thermally stable and have a large Kerr rotation. Thus $\mathrm{MnBiAl}$ may be a good candidate for magneto-optical recording applications.

\section{ACKNOWLEDGMENTS}

We are grateful to the National Science Foundation for financial support under Grants No. DMR-8918889 and No. INT-8715441, and we thank the Research Corporation for their financial support of this project. We also thank Dr. S. S. Jaswal, Dr. Z . S. Shan, and D. Wang for helpful discussions.

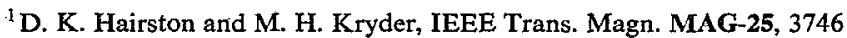
(1989).

${ }^{2}$ F. J. A. M. Greidanus, W. B. Zeper, F. J. A. de Broeder, W. F. Godlieb, and P. F. Carcia, Appl. Phys. Lett. 54, 2481 (1989).

${ }^{3}$ K. Egashira and T. Yamada, J. Appl. Phys. 45, 3643 (1974).

${ }^{4}$ T. Katayama, Y. Suzuki, H. Awano, Y. Nishihara, and N. Koshizuka, Phys. Rev. Lett. 60, 1426 (1988).

${ }^{5}$ J. Schoenes and W. Reim, J. Magn. Magn. Mater. 54-57, 1371 (1986).

${ }^{6}$ M. Naoe, N. Kitamura, M. Shoji, and A. Nagai, J. Appl. Phys. 63, 3663 (1988).

${ }^{7}$ D. Chen and Y. Gondo, J. Appl. Phys. 35, 1024 (1964).

${ }^{8}$ W. K. Unger, E. Wolfgang, H. Harms, and H. Haudek, J. Appl. Phys. 43, 2875 (1972).

${ }^{9}$ A. Katsui, A. Shibukawa, H. Terui, and K. Egashira, J. Appl. Phys. 47, 5069 (1976).

${ }^{10}$ A. Katsui, J. Appl. Phys. 47, 4663 (1976).

${ }^{11}$ Y. J. Wang, J. Magn. Magn. Mater. 84, 39 (1990).

${ }^{12}$ J. X. Shen, R. D. Kirby, and D. J. Sellmyer, J. Magn. Magn. Mater. 81, 107 (1989).

${ }^{13}$ R. Coehoorn and R. A. de Groot, J. Phys. F 15, 2135 (1985).

${ }^{14}$ D. Chen, Y. Gondo, and M. D. Blue, J. Appl. Phys, 36, 1261 (1965). 